\title{
Changes in microRNA expression associated with metastasis and survival in patients with uveal melanoma
}

\author{
Ayushi Vashishtha ${ }^{1}$, Tae Jin Lee ${ }^{1}$, Ashok Sharma ${ }^{1}$ and John J. Wallbillich ${ }^{1,2,3}$ \\ ${ }^{1}$ Center for Biotechnology and Genomic Medicine, Medical College of Georgia, Augusta University, Augusta, GA, USA \\ ${ }^{2}$ Department of Obstetrics and Gynecology, Medical College of Georgia, Augusta University, Augusta, GA, USA \\ ${ }^{3}$ Current address: Department of Oncology, Wayne State University College of Medicine, Detroit, MI, USA \\ Correspondence to: John J. Wallbillich, email: jwallbil@med.wayne.edu
}

Keywords: uveal melanoma; metastasis; survival; microRNA; biomarker

Received: December 21, 2019 Accepted: March 19, 2020

Published: April 21, 2020

Copyright: Vashishtha et al. This is an open-access article distributed under the terms of the Creative Commons Attribution License 3.0 (CC BY 3.0), which permits unrestricted use, distribution, and reproduction in any medium, provided the original author and source are credited.

\section{ABSTRACT}

Uveal melanoma (UM) is a major intraocular cancer that is molecularly distinct from cutaneous melanoma. Approximately half of patients with UM eventually develop metastasis. The prognosis of metastatic UM is poor, with a median overall survival (OS) of less than a year. In this study, we sought to identify microRNAs (miRNAs) associated with metastasis and OS in UM. We analyzed the miRNA expression and clinical outcomes data from The Cancer Genome Atlas (TCGA) dataset for UM. Differential expression analyses were conducted for each miRNA with respect everdevelopment of metastasis. Multiple survival analyses were done, using the Cox proportional hazards model, to evaluate interactions between miRNA expression, metastasis, and OS. A total of 22 miRNAs ( 3 upregulated and 19 downregulated) were differentially expressed between patients with vs. without metastatic UM. These 22 miRNAs could be grouped into four clusters based on similarities in expression patterns. Of the 22 miRNAs differentially expressed with respect to metastasis, 21 were significantly associated with OS. The expression of multiple miRNAs was significantly associated with metastasis and overall survival in patients with UM. Further investigation of these miRNAs as biomarkers and/or therapeutic targets is warranted in the push to improve outcomes for patients with metastatic UM.

\section{INTRODUCTION}

Uveal melanoma (UM) is the most common primary intraocular cancer occurring in adults $[1,2]$. The mortality of UM patients is approximately $40-50 \%$; the leading contributor to mortality is development of metastasis, which occurs in up to $50 \%$ of UM patients [3,4]. For patients with metastatic UM, the 1-year survival rate is $20 \%$, the 5 -year survival rate is less than $5 \%$, and the median overall survival is only $6-12$ months $[1,5-8]$. There is no effective therapeutic intervention to treat metastatic UM [4].

While UM may bear histologic resemblance to its more-common cutaneous counterpart, it is considered molecularly distinct. In contrast to cutaneous melanoma, UM has a lower mutational burden [9] and lacks characteristic BRAF and NRAS mutations $[9,10]$. Rather, most UM tumors contain GNAQ or GNA11 mutations
[11], MAPK pathway activations [12], and cytogenetic abnormalities (monosomy 3 and trisomy 8q) [13].

Given the biologic uniqueness of UM, its propensity to metastasize, and the poor survival outcomes and lack of adequate treatment for metastatic UM, there is a great need to uncover the molecular mechanisms of metastasis in UM and to discover predictive and prognostic biomarkers so as to better optimize understanding and management of this challenging oncologic condition.

Several genetic mutations and gene expression alterations have been associated with the molecular mechanisms responsible for the progression of UM [14]. It has also been shown that epigenetic modifications including microRNAs (miRNAs) are associated with the pathology and progression of UM [14]. miRNAs are small, non-coding RNAs approximately 22 nucleotides in length. Each microRNA can play a crucial role in regulating the expression of multiple genes. miRNAs typically regulate 
gene expression by altering mRNA stability or repressing translation of mRNA to protein. Several miRNAs play an important role (tumor suppressors or tumor promoters) in cancer development and progression including metastasis [15-20]. However, data are limited regarding the role of miRNAs in metastatic UM [21-23]. Therefore, the purpose of this study was to identify miRNAs associated with UM metastasis and overall patient survival.

\section{RESULTS}

\section{Demographics, key clinical data, and differences in OS for patients with metastatic vs. non- metastatic UM}

Profiling data from 1,598 miRNAs was available for primary-site tumor samples from 80 patients with UM in TCGA. Of those patients, $50(62.5 \%)$ had never developed metastasis, while $30(37.5 \%)$ had ever developed metastasis. Key demographic and clinical data for UM patients with metastatic and non-metastatic UM were identified and compared across the two groups. Compared to those with non-metastatic UM, patients with metastatic UM more often had epithelioid histology, less often had spindle cell histology, and had a higher proportion of advanced (stage III-IV) disease at initial diagnosis ( $p$-values all $<0.05)$. There was no significant difference between non-metastatic and metastatic UM with respect to age, gender, or tumor thickness (Table 1). Survival analysis for metastatic vs. non-metastatic UM showed a major difference (hazard ratio $[\mathrm{HR}]=15.24$; $p$-value $=$ $2.42 \times 10^{-4}$ ) with respect to overall survival (OS) between those groups (Figure 1A).

\section{miRNAs differentially expressed in UM with respect to metastasis}

To investigate the miRNAs associated with UM metastasis, we analyzed the expression of the 1,598 miRNAs available in TCGA UM dataset. Differential expression analysis was done for each of those 1,598 miRNAs between UM patients with $(n=30)$ and without $(n=50)$ metastasis. The volcano plot depicting the results of this differential expression analysis is shown in Figure 1B. In total, we discovered 76 miRNAs (24 upregulated and 52 downregulated) significantly (adj. $p$-value $<0.05$ ) dysregulated in patients with metastasis as compared to patients without metastasis (Supplementary Table 1). The 22 most significantly ( $>2$-fold change and adj. $p$-value $<0.01$ ) dysregulated miRNAs in patients with metastatic UM are listed in Table 2. A heatmap depicting the expression levels of these 22 miRNAs in individual UM patients is shown in Figure 2A. Out of the 22 miRNAs, 3 are upregulated and 19 are downregulated in patients with metastasis. The upregulated miRNAs in patients with metastasis are miR-199a-5p (3.29-fold), miR-708-5p (2.29-fold), and miR-592 (2.05-fold). Similarly, the most downregulated miRNAs in patients with metastasis are miR-508-3p (0.06-fold), miR-509-3p (0.07-fold), miR508-5p (0.08-fold), miR-514a-3p (0.08-fold), miR-506$3 p$ (0.11-fold), miR-509-3-5p (0.12-fold), miR-513c-5p (0.14-fold), miR-513a-5p (0.17-fold), and miR-513b-5p (0.24-fold) as shown in Figure 3.

\section{Cluster analysis of miRNAs differentially expressed in UM metastasis}

A pairwise correlation was computed for the 22 miRNAs significantly differentially expressed in patients with metastasis. The cluster analysis of the correlation matrix revealed 4 clusters of highly correlated miRNAs (Figure 2B). This analysis provides us the groups of miRNAs which are co-regulated in metastatic UM. Cluster-1 included 9 downregulated miRNAs including miR-508-3p, miR-509-3p, miR-508-5p, miR-514a-3p, miR-506-3p, miR-509-3-5p, miR-513c-5p, miR-513a-5p, and miR-513b-5p. These 9 miRNAs have highly correlated expression levels (average correlation coefficient $=0.95$ ), indicating a probable common regulatory mechanism. Cluster-2 included 2 downregulated miRNAs: miR-221$3 p$ and miR-222-3p. The correlation coefficient between the expression of these miRNAs was 0.92 . The Cluster-3 included 5 miRNAs (miR-125b-5p, miR-125b-2-3p, let7c-3p, miR-140-5p, and miR-181b-5p), which are also downregulated in the patients with metastasis, indicating that these miRNAs have a protective role in UM. Cluster-4 included miR-592, miR-708-5p, and miR-199a-5p, and these 3 miRNAs are upregulated in UM, therefore the miRNAs of this cluster have a negative correlation with the miRNAs of the other three clusters.

\section{miRNAs associated with survival status in UM}

Cox proportional hazard analysis found that 64 miRNAs were significantly (adj. $p$-value $<0.001$ and $\mathrm{HR}>4$ or $\mathrm{HR}<0.2$ ) correlated with patient survival (Supplementary Table 2). The 15 miRNAs most significantly associated with overall survival (OS) of patients $(\mathrm{HR}>10$ or HR $<0.10)$ are listed in Table 3; the Kaplan-Meier OS curves of these miRNAs are shown in Figure 4. Of the 22 miRNAs significantly differentially expressed in patients with metastasis, 21 were significantly $(p<0.05)$ associated with OS.

\section{Target genes and pathways regulated by miRNAs associated with UM metastasis}

A comprehensive search of experimentally validated target genes regulated by miRNAs found to be associated with UM metastasis was performed using Ingenuity Pathway Analysis software (QIAGEN, Redwood City, CA, USA). Further bioinformatics analyses 
Table 1: Demographics and key clinical data for patients with non-metastatic vs. metastatic UM

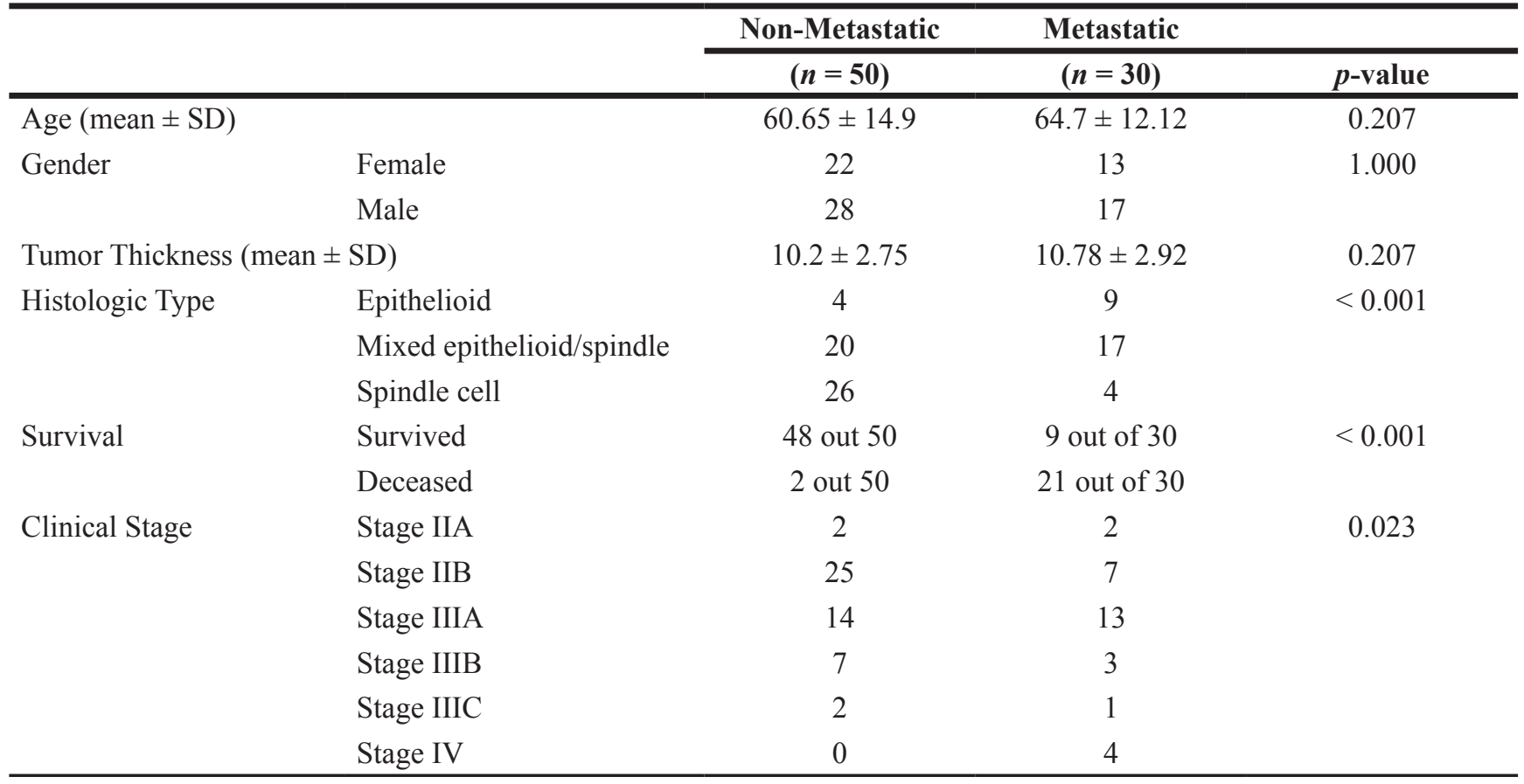

were performed to discover pathways and biological processes associated with these target genes. The top canonical pathways associated with these genes include p53 signaling, regulation of epithelial-mesenchymal transition pathway, cell cycle G1/S checkpoint regulation, ILK signaling, and PTEN signaling (Table 4). The top biological functions include apoptosis, necrosis, growth of tumor, cell proliferation, invasion, movement, migration, and cell cycle progression (Table 4). We also performed network analysis to discover the interactions between the target genes. The top-scoring network is shown in Figure 5. The network analysis revealed that the hub genes of the network are $M Y C, V I M, A R, E R B B 2, H I F 1 A, F O S$, $K R A S, V E G F, P K A, E L A V L 1$, and GSK3B. These hub genes interact with many $(>15)$ nodes on the interaction network and are likely important for gene expression dynamics (mechanism). The top upstream regulators of the target genes are TP53, EGF, TGFB1, PTEN, MYC,
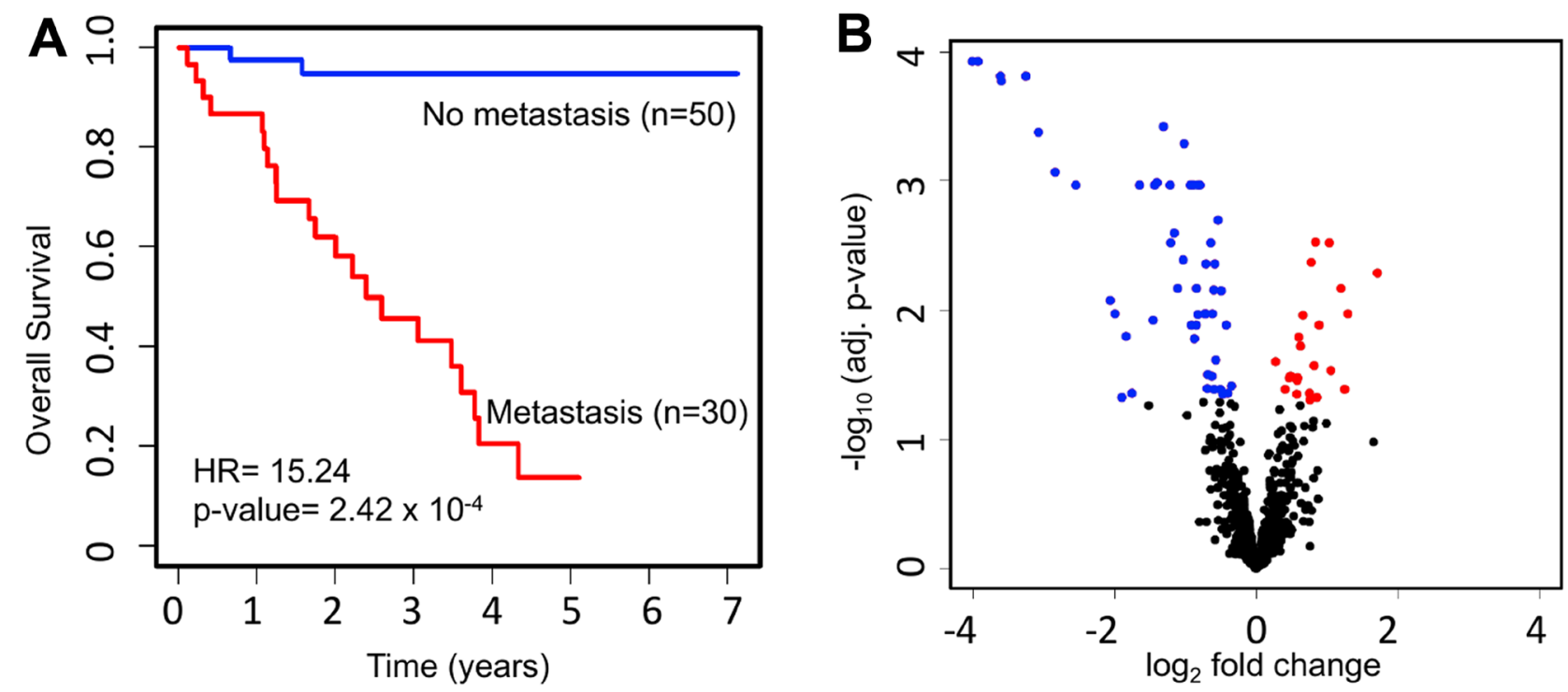

Figure 1: Survival and miRNA expression differences between patients who ever vs. never developed metastatic UM. (A) Kaplan-Meier survival curves showing a major difference in OS $\left(\mathrm{HR}=15.24\right.$; $p$-value $\left.=2.42 \times 10^{-4}\right)$ for UM patients with $(n=30)$ vs. without $(n=50)$ metastasis. (B) Volcano plot depicting differentially expressed miRNAs. The expression level of 1,598 miRNAs was compared between UM patients with metastasis $(n=30)$ and without metastasis $(n=50)$. In total, we discovered 76 significantly dysregulated miRNAs in metastatic patients, including 24 upregulated (red) and 52 downregulated (blue). 
Table 2: The 22 most significantly dysregulated miRNAs in patients with metastatic UM

\begin{tabular}{|c|c|c|c|c|c|c|}
\hline miRNA accession ID & miRNA target name & Fold- change & Adj. $p$-value & HR & Adj. $p$-value & Concordance \\
\hline \multicolumn{7}{|c|}{ Down-regulated miRNAs } \\
\hline MIMAT0002880 & hsa-miR-508-3p & 0.062 & $1.19 \times 10^{-4}$ & 0.08 & $4.77 \times 10^{-7}$ & 0.780 \\
\hline MIMAT0002881 & hsa-miR-509-3p & 0.065 & $1.19 \times 10^{-4}$ & 0.10 & $3.25 \times 10^{-6}$ & 0.766 \\
\hline MIMAT0004778 & hsa-miR-508-5p & 0.081 & $1.56 \times 10^{-4}$ & 0.10 & $2.27 \times 10^{-6}$ & 0.770 \\
\hline MIMAT0002883 & hsa-miR-514a-3p & 0.082 & $1.69 \times 10^{-4}$ & 0.09 & $6.38 \times 10^{-7}$ & 0.778 \\
\hline MIMAT0002878 & hsa-miR-506-3p & 0.105 & $1.56 \times 10^{-4}$ & 0.10 & $4.88 \times 10^{-6}$ & 0.760 \\
\hline MIMAT0004975 & hsa-miR-509-3-5p & 0.118 & $4.20 \times 10^{-4}$ & 0.11 & $1.57 \times 10^{-5}$ & 0.747 \\
\hline МIMAT0005789 & hsa-miR-513c-5p & 0.139 & $8.61 \times 10^{-4}$ & 0.11 & $8.88 \times 10^{-6}$ & 0.752 \\
\hline MIMAT0002877 & hsa-miR-513a-5p & 0.171 & $1.09 \times 10^{-3}$ & 0.09 & $1.10 \times 10^{-5}$ & 0.727 \\
\hline MIMAT0005788 & hsa-miR-513b-5p & 0.239 & $8.55 \times 10^{-3}$ & 0.07 & $8.42 \times 10^{-5}$ & 0.688 \\
\hline MIMAT0000278 & hsa-miR-221-3p & 0.319 & $1.09 \times 10^{-3}$ & 0.39 & 0.039 & 0.607 \\
\hline МIMAT0000279 & hsa-miR-222-3p & 0.370 & $1.09 \times 10^{-3}$ & 0.28 & $5.09 \times 10^{-3}$ & 0.645 \\
\hline MIMAT0000097 & hsa-miR-99a-5p & 0.378 & $1.03 \times 10^{-3}$ & 0.24 & $1.22 \times 10^{-3}$ & 0.713 \\
\hline MIMAT0000064 & hsa-let-7c-5p & 0.403 & $3.83 \times 10^{-4}$ & 0.27 & $3.19 \times 10^{-3}$ & 0.694 \\
\hline МIMAT0005924 & hsa-miR-1270 & 0.430 & $1.09 \times 10^{-3}$ & 0.17 & $1.82 \times 10^{-4}$ & 0.704 \\
\hline MIMAT0000423 & hsa-miR-125b-5p & 0.433 & $3.03 \times 10^{-3}$ & 0.24 & $1.31 \times 10^{-3}$ & 0.711 \\
\hline MIMAT0004603 & hsa-miR-125b-2-3p & 0.447 & $2.56 \times 10^{-3}$ & 0.46 & 0.075 & 0.641 \\
\hline MIMAT0026472 & hsa-let-7c-3p & 0.464 & $6.82 \times 10^{-3}$ & 0.28 & $3.84 \times 10^{-3}$ & 0.693 \\
\hline MIMAT0000431 & hsa-miR-140-5p & 0.488 & $4.13 \times 10^{-3}$ & 0.15 & $7.81 \times 10^{-5}$ & 0.685 \\
\hline MIMAT0000257 & hsa-miR-181b-5p & 0.494 & $5.17 \times 10^{-4}$ & 0.14 & $3.46 \times 10^{-5}$ & 0.718 \\
\hline \multicolumn{7}{|l|}{ Up-regulated miRNAs } \\
\hline MIMAT0003260 & hsa-miR-592 & 2.047 & $3.02 \times 10^{-3}$ & 4.71 & $4.49 \times 10^{-4}$ & 0.723 \\
\hline MIMAT0004926 & hsa-miR-708-5p & 2.292 & $6.82 \times 10^{-3}$ & 4.55 & $6.28 \times 10^{-4}$ & 0.682 \\
\hline MIMAT0000231 & hsa-miR-199a-5p & 3.286 & $5.15 \times 10^{-3}$ & 5.50 & $1.95 \times 10^{-4}$ & 0.669 \\
\hline
\end{tabular}

SP1, ERBB2, FGF2, HGF, TP63, ESR1, E2F1, KRAS, and $P I 3 K$ complex (Table 4). These upstream regulators are the predicted transcriptional regulators in the pathway.

\section{DISCUSSION}

The development of metastases plays an important role in UM patient prognosis. Molecular biomarkers associated with UM metastasis may help in accurately identifying high-risk patients and in discovering potential therapeutic targets for metastatic UM treatment. MicroRNAs are small single-stranded endogenous noncoding RNAs, which are involved in the posttranscriptional regulation of expression of their targeted mRNAs. It has been established that aberrant expression of miRNAs leads to progression and metastasis of several cancers. In the past decade, several studies have examined the role of microRNAs in pathogenesis and progression of UM by utilizing plasma [24], serum [25], cell lines [26-29], and clinical tissue specimens [21-23, 30]. Uveal melanoma is a very rare cancer, making it difficult to obtain a large number of samples from a single institution. TCGA is a landmark cancer genomics dataset which has molecularly characterized cancer and matched normal samples and is an especially important resource for rare cancers such as UM. TCGA also provides data on various clinical and demographic parameters associated with UM and analyzes this larger sample set with careful experimental design and proper control groups. The use of this larger sample size increases the statistical power of the analysis. Also, to minimize the false positives, we have used a very stringent cutoff to select differentially expressed miRNAs. This approach enabled us to identify several novel miRNAs potentially related to UM metastasis which may have clinical, biological, or mechanistic relevance to UM and may expand our understanding of UM tumor progression.

In this study, we found 22 miRNAs highly dysregulated ( $>2$-fold change and $p<0.01)$ in UM patients with (vs. without) ever-development of metastasis. $21(95 \%)$ of those miRNAs associated with metastasis were also significantly associated with poor OS. The 22 miRNAs associated with metastasis could be divided into four distinct clusters based on highly correlated expression patterns within each cluster.

Cluster-1 included the 9 most downregulated miRNAs including miR-508-3p, miR-509-3p, miR-5085p, miR-514a-3p, miR-506-3p, miR-509-3-5p, miR513c-5p, miR-513a-5p, and miR-513b-5p. For miR508-3p, a recent study showed that decreased expression 
was significantly associated with metastasis, while overexpression suppressed the epithelial-mesenchymal transition process, in patients with triple-negative breast cancer [31]. Another study found that miR-508-3p and
miR-509-3p were downregulated in renal cell carcinoma tissues, while overexpression of those miRNAs suppressed renal cell carcinoma proliferation, invasion, and migration in vitro [32]. In ovarian cancer patients, increased

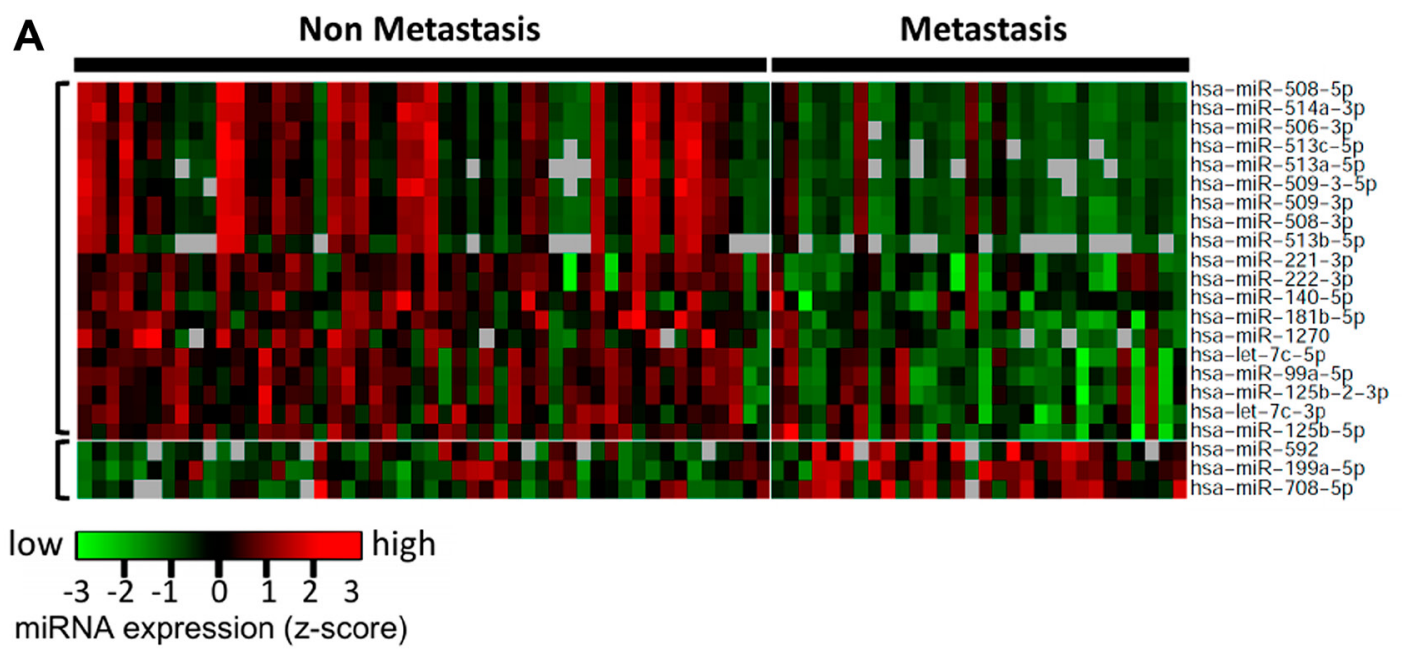

B
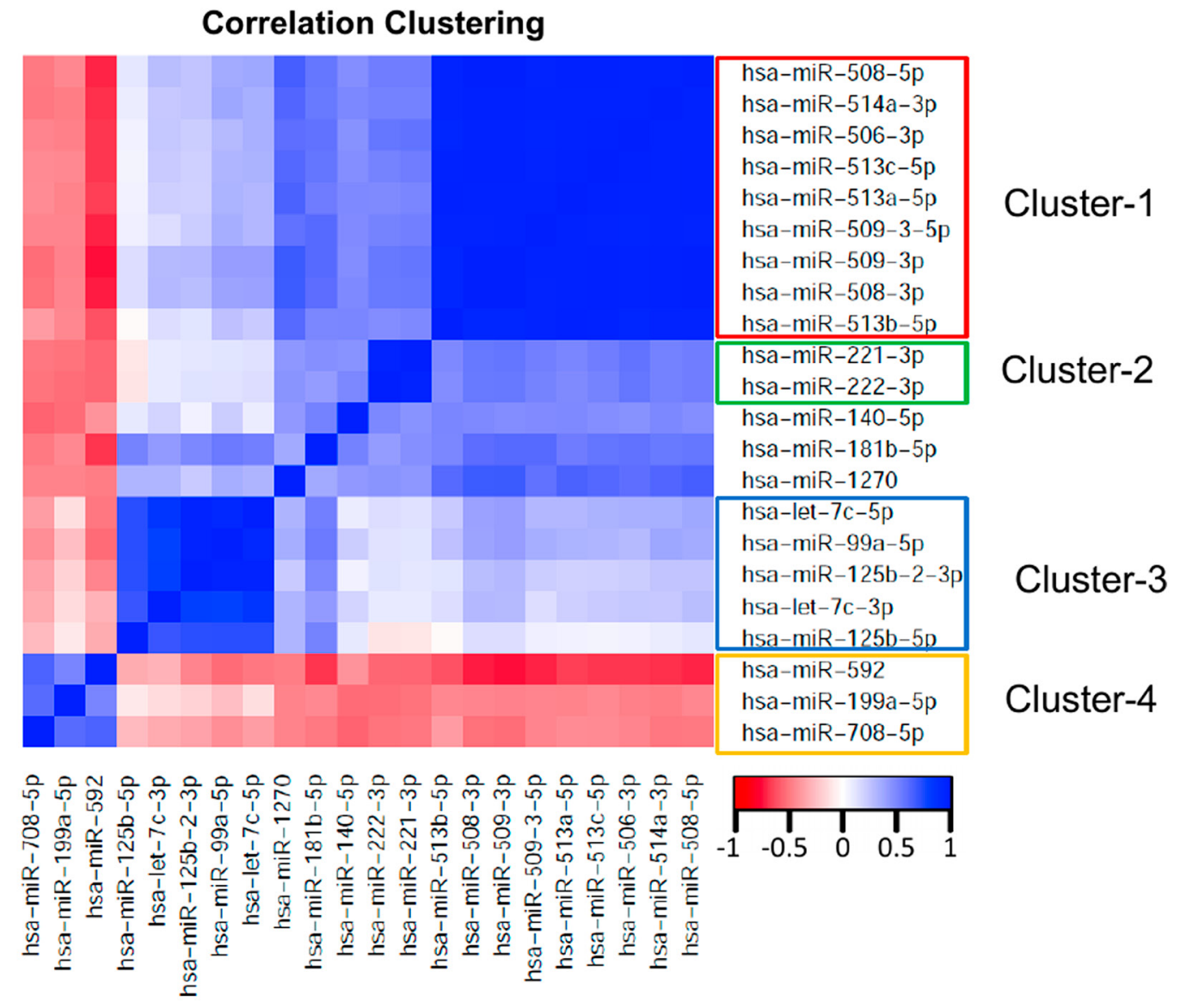

Figure 2: Heatmap and correlation clustering of the miRNAs significantly differentially expressed with respect to ever-development of metastatic UM. (A) Heatmap representing the expression of the 22 most highly dysregulated miRNAs in UM metastasis. Out of these 22 miRNAs, 3 are upregulated and 19 are downregulated in patients with metastasis. Each column represents one patient and rows represent miRNAs. (B) Pairwise correlations for the 22 miRNAs significantly differentially expressed in UM patients with metastasis. The cluster analysis of the correlation matrix revealed 4 major clusters of highly correlated miRNAs. Cluster-1 included 9 downregulated miRNAs, Cluster-2 included 2 downregulated miRNAs, Cluster-3 included 5 downregulated miRNAs, and Cluster-4 included the 3 upregulated miRNAs. 
expression of miR-508-3p, miR-508-5p, miR-509-3p, and miR-508-5p was correlated with improved clinical outcomes [33]. Data from our study suggests that this miRNA cluster functions as a tumor suppressor as it is markedly downregulated in UM patients with metastasis. Further, the highly correlated expression levels (average correlation coefficient $=0.95$ ) of these 9 miRNAs suggests a probable common regulatory mechanism. Therefore,
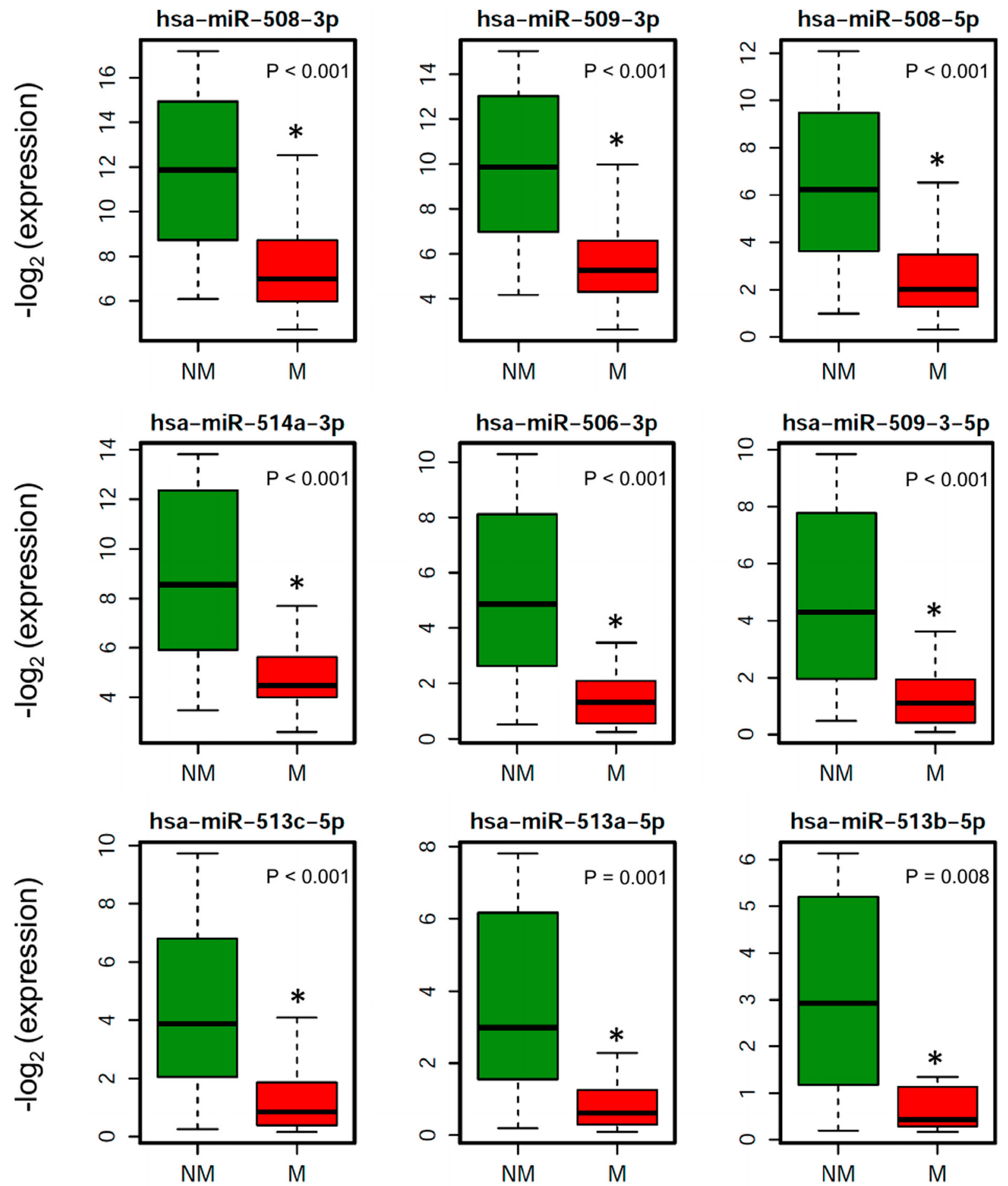

NM: Non-metastasis

M: Metastasis

Figure 3: Boxplots showing the distribution of the miRNA expression of the 9 most downregulated miRNAs in patients who ever developed metastasis compared to patients without metastasis. 


\begin{tabular}{|c|c|c|c|c|c|c|}
\hline miRNA accession ID & miRNA target name & HR & Adj. $p$-value & Concordance & Fold change & Adj. $p$-value \\
\hline \multicolumn{7}{|l|}{$\begin{array}{l}\text { miRNAs with Hazard } \\
\text { Ratio }<0.10\end{array}$} \\
\hline MIMAT0022717 & hsa-miR-873-3p & 0.041 & $9.73 \times 10^{-7}$ & 0.721 & 0.66 & 0.107 \\
\hline MIMAT0015087 & hsa-miR-514b-5p & 0.062 & $2.71 \times 10^{-5}$ & 0.702 & 0.25 & 0.011 \\
\hline MIMAT0005788 & hsa-miR-513b-5p & 0.070 & $8.42 \times 10^{-5}$ & 0.688 & 0.24 & 0.009 \\
\hline MIMAT0022702 & hsa-miR-514a-5p & 0.071 & $9.56 \times 10^{-5}$ & 0.686 & 0.27 & 0.048 \\
\hline MIMAT0002879 & hsa-miR-507 & 0.082 & $2.70 \times 10^{-4}$ & 0.676 & 0.30 & 0.045 \\
\hline MIMAT0002880 & hsa-miR-508-3p & 0.083 & $4.77 \times 10^{-7}$ & 0.780 & 0.06 & 0.0001 \\
\hline MIMAT0002883 & hsa-miR-514a-3p & 0.085 & $6.38 \times 10^{-7}$ & 0.778 & 0.08 & 0.0002 \\
\hline MIMAT0015020 & hsa-miR-548v & 0.087 & $1.19 \times 10^{-5}$ & 0.715 & 0.53 & 0.013 \\
\hline MIMAT0002877 & hsa-miR-513a-5p & 0.087 & $1.10 \times 10^{-5}$ & 0.727 & 0.17 & 0.001 \\
\hline MIMAT0004778 & hsa-miR-508-5p & 0.096 & $2.27 \times 10^{-6}$ & 0.770 & 0.08 & 0.0002 \\
\hline MIMAT0002881 & hsa-miR-509-3p & 0.099 & $3.25 \times 10^{-6}$ & 0.766 & 0.07 & 0.0001 \\
\hline \multicolumn{7}{|c|}{ miRNAs with Hazard Ratio $>10$} \\
\hline MIMAT0000269 & hsa-miR-212-3p & 17.126 & $3.64 \times 10^{-7}$ & 0.715 & 1.68 & 0.045 \\
\hline MIMAT0001635 & hsa-miR-452-5p & 11.806 & $1.32 \times 10^{-6}$ & 0.729 & 1.60 & 0.079 \\
\hline MIMAT0004514 & hsa-miR-29b-1-5p & 10.898 & $3.80 \times 10^{-6}$ & 0.681 & 1.49 & 0.045 \\
\hline MIMAT0004482 & hsa-let-7b-3p & 10.184 & $3.42 \times 10^{-6}$ & 0.753 & 1.79 & 0.003 \\
\hline
\end{tabular}

these miRNAs as well as their target genes may have therapeutic potential to inhibit tumor metastasis and progression.

Cluster-2 included 2 miRNAs, miR-221-3p and miR-222-3p, which were both downregulated in UM patients with metastasis. In a recent study, miR-221$3 p$ and miR-222-3p were also downregulated in gastric cancer cells with high metastatic potential [18].

Cluster- 3 contained 5 downregulated miRNAs with respect to metastasis in UM: miR-125b-5p, miR-125b2-3p, let-7c-3p, miR-140-5p, and miR-181b-5p. Similar to our findings, a recent study also found that miR-140$5 p$ was abnormally downregulated in melanoma tissues and cells [23]. Overexpression of miR-125b-5p inhibited cell proliferation, migration, and invasion in esophageal squamous cell carcinoma [17]. Five hub miRNAs including miR-125b-5p, miR-145-5p, let-7c-5p, miR-218$5 p$, and miR-125b-2-3p were also found to be related to the prognosis of colorectal cancer [15]. Of these miRNAs, miR-125b-5p and miR-125b-2-3p were also significantly associated in our study as part of the third cluster.

Cluser-4 included the three most significantly upregulated miRNAs, including miR-592, miR-708-5p, and miR-199a-5p. Previous studies have also shown that miR199a regulates melanoma metastasis related genes and may provide new therapeutic targets $[19,21]$. In a recent study, higher expression of miRNA 199a was observed in UM with liver metastasis [22]. Using a genome-wide microarray based approach, another study found that expression of miRNA199a was one of the most significant discriminators of low metastasis and high metastasis risk of UM patients [21].

The additional bioinformatic analyses we performed identified the target genes and pathways regulated by the
miRNAs found to be associated with UM. Several key transcription regulators (TP53, MYC, SP1, TP63, E2F1), growth factors (EGF, TGFB1, FGF2, HGF) and other key regulators including PTEN, ERBB2, ESR1, KRAS and PI3K-complex were found as key targets using this analysis. Previous studies have also reported constitutive activation of these oncogenic pathways in primary UM $[34,35]$. Biological functions related to metastasis including cell cycle progression, cell proliferation, invasion, movement and migration were significantly enriched in the target genes.

With the recent advancement of molecular technologies, miRNAs have newfound potential to serve as viable therapeutic tools. Molecular approaches such as AMOs (anti-miR oligonucleotides), LNA anti-miRs, antagomirs, miRNA sponges, and S-miRs (small molecule inhibitors to target specific miRNAs) are available to inhibit the miRNAs overexpressed in cancer [36-40]. On the other hand, molecular approaches to restore the decreased expression of miRNAs downregulated in cancer are also available and include miRNA mimics (double-stranded synthetic RNAs that mimic endogenous miRNAs) and miRNA expression vectors. Several studies have used miRNA replacement therapy in experimental models [41-43]. Additionally, in a recent study, aptamermiRNA conjugates were used as a novel tool for targeted delivery of miRNAs [44]. Several miRNA-based therapies are already in clinical trials, for example, miR-16 mimics are under phase 1 clinical trials for patients with recurrent thoracic cancer [45].

A major limitation of this study was a lack of experimental validation of the findings with either a separate dataset from patient samples or through in vivo or 
in vitro experiments. Further, as with other investigations primarily based on data from TCGA, our analyses used retrospectively obtained data and TCGA UM patient population may not be fully generalizable to some UM patient populations with demographic or clinical features under-represented in TCGA.

In conclusion, this study identified, in primarysite tumor samples, altered miRNA expression patterns associated with ever-development of metastasis in patients with uveal melanoma. We found several known tumor suppressor miRNAs to be downregulated in UM patients with metastasis. These results support the increasingly accepted concept that miRNAs play a major role in metastasis. Our finding of $95 \%$ overlap between (a) miRNAs associated with UM metastasis and (b) miRNAs associated with poor survival in patients with UM warrants further investigation those overlapping miRNAs. Future
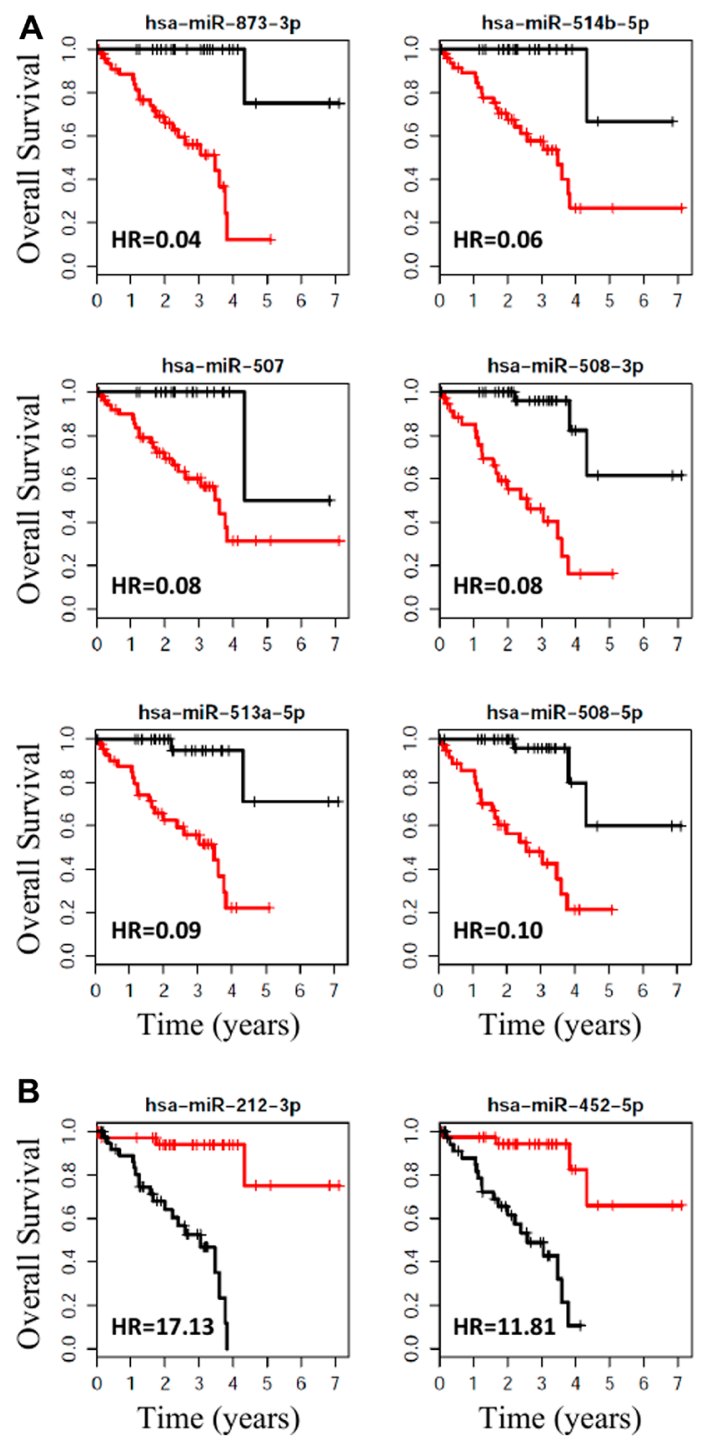

evaluation of the 21 overlapping miRNA as prognostic biomarkers and/or therapeutic targets may be a step toward improved outcomes for those with metastatic UM, a patient population that suffers from high mortality and a lack of effective treatment options.

\section{MATERIALS AND METHODS}

\section{Dataset}

The Cancer Genome Atlas (TCGA; RRID: SCR_003193) is one of the foremost data repositories providing molecular characterization of more than 20,000 primary cancers, including unprecedented amounts of miRNA sequence data ( 11,000 libraries) across 33 cancer types [20]. We therefore chose TCGA as the dataset for this investigation. We utilized this high-quality data for
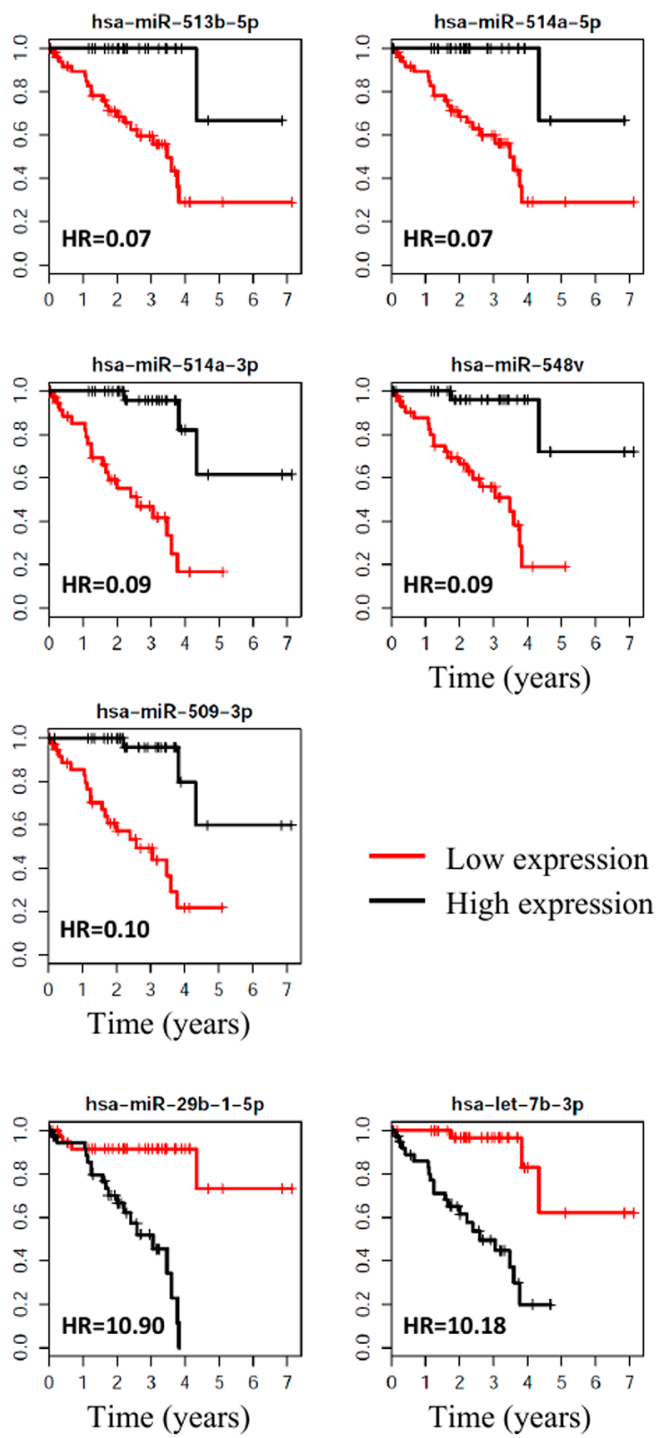

Figure 4: Survival plots, with respect to high vs. low expression, for the miRNAs associated with metastasis that were the most significantly associated with OS for UM. (A) The 11 miRNAs that were most $(\mathrm{HR}<0.10)$ protective for OS when highly expressed. (B) The 4 miRNAs that were associated with the highest (Hazard Ratio $>10$ ) risk of death. 
Table 4: Top canonical pathways, biological functions and upstream regulators

\begin{tabular}{|c|c|c|}
\hline \multicolumn{2}{|l|}{ Canonical pathways } & $p$-value \\
\hline \multicolumn{2}{|c|}{ 1. Molecular Mechanisms of Cancer } & $1.58 \times 10^{-19}$ \\
\hline \multicolumn{2}{|c|}{ 2. Senescence Pathway } & $2.51 \times 10^{-18}$ \\
\hline \multicolumn{2}{|l|}{ 3. p53 Signaling } & $1.00 \times 10^{-16}$ \\
\hline \multicolumn{2}{|c|}{ 4. Pancreatic Adenocarcinoma Signaling } & $1.26 \times 10^{-14}$ \\
\hline \multicolumn{2}{|c|}{ 5. Glioblastoma Multiforme Signaling } & $1.00 \times 10^{-12}$ \\
\hline \multicolumn{2}{|c|}{ 6. Aryl Hydrocarbon Receptor Signaling } & $3.16 \times 10^{-12}$ \\
\hline \multicolumn{2}{|c|}{ 7. Regulation of the Epithelial-Mesenchymal Transition Pathway } & $3.16 \times 10^{-12}$ \\
\hline \multicolumn{2}{|c|}{ 8. Chronic Myeloid Leukemia Signaling } & $6.30 \times 10^{-12}$ \\
\hline \multicolumn{2}{|c|}{ 9. Cell Cycle: G1/S Checkpoint Regulation } & $7.94 \times 10^{-12}$ \\
\hline \multicolumn{2}{|l|}{ 10. ILK Signaling } & $1.99 \times 10^{-11}$ \\
\hline \multicolumn{2}{|l|}{ 11. PTEN Signaling } & $2.51 \times 10^{-11}$ \\
\hline \multicolumn{2}{|c|}{ 12. Colorectal Cancer Metastasis Signaling } & $3.98 \times 10^{-11}$ \\
\hline \multicolumn{2}{|l|}{ 13. IL-8 Signaling } & $5.01 \times 10^{-11}$ \\
\hline \multicolumn{2}{|c|}{ 14. Glucocorticoid Receptor Signaling } & $1.94 \times 10^{-10}$ \\
\hline \multicolumn{2}{|c|}{ 15. Cyclins and Cell Cycle Regulation } & $1.58 \times 10^{-09}$ \\
\hline \multicolumn{2}{|c|}{ Diseases or functions annotation } & p-value \\
\hline \multicolumn{2}{|l|}{ 1. Apoptosis } & $1.43 \times 10^{-37}$ \\
\hline \multicolumn{2}{|l|}{ 2. Necrosis } & $1.54 \times 10^{-37}$ \\
\hline \multicolumn{2}{|l|}{ 3. Growth of tumor } & $1.65 \times 10^{-37}$ \\
\hline \multicolumn{2}{|c|}{ 4. Cell proliferation of tumor cell lines } & $1.89 \times 10^{-37}$ \\
\hline \multicolumn{2}{|l|}{ 5. Invasion of cells } & $2.06 \times 10^{-35}$ \\
\hline \multicolumn{2}{|c|}{ 6. Cell movement of tumor cell lines } & $3.85 \times 10^{-34}$ \\
\hline \multicolumn{2}{|l|}{ 7. Migration of cells } & $1.55 \times 10^{-32}$ \\
\hline \multicolumn{2}{|c|}{ 8. Growth of malignant tumor } & $1.76 \times 10^{-32}$ \\
\hline \multicolumn{2}{|l|}{ 9. Cell movement } & $7.82 \times 10^{-32}$ \\
\hline 10. Migration of tum & & $9.59 \times 10^{-32}$ \\
\hline 11. Cell cycle progre & & $1.68 \times 10^{-31}$ \\
\hline 12. Invasion of tumo & & $1.97 \times 10^{-31}$ \\
\hline Upstream regulator & Molecule type & p-value \\
\hline 1. $T P 53$ & transcription regulator & $2.21 \times 10^{-31}$ \\
\hline 2. $E G F$ & growth factor & $4.00 \times 10^{-30}$ \\
\hline 3. $T G F B 1$ & growth factor & $5.81 \times 10^{-30}$ \\
\hline 4. PTEN & phosphatase & $1.18 \times 10^{-29}$ \\
\hline 5. $M Y C$ & transcription regulator & $2.56 \times 10^{-28}$ \\
\hline 6. $S P 1$ & transcription regulator & $4.87 \times 10^{-27}$ \\
\hline 7. $E R B B 2$ & kinase & $2.54 \times 10^{-26}$ \\
\hline 8. FGF2 & growth factor & $9.69 \times 10^{-26}$ \\
\hline 9. $H G F$ & growth factor & $1.66 \times 10^{-24}$ \\
\hline 10. TP63 & transcription regulator & $3.09 \times 10^{-23}$ \\
\hline 11. ESR1 & ligand-dependent nuclear receptor & $4.44 \times 10^{-23}$ \\
\hline 12. $E 2 F 1$ & transcription regulator & $6.48 \times 10^{-23}$ \\
\hline 13. KRAS & enzyme & $4.26 \times 10^{-22}$ \\
\hline 14. PI3K (complex) & complex & $5.69 \times 10^{-22}$ \\
\hline
\end{tabular}

our study to analyze the differential miRNA expression between patients with and without metastasis. For the sake of this analysis, "metastasis" refers to metastasis at either initial presentation/diagnosis or recurrence (i. e., everdevelopment of metastasis).
TCGA miRNA expression data was generated using the Illumina HiSeq/GA miRseq and was reported as counts normalized to reads per million mapped reads (RPM). The uveal melanoma miRNA dataset was downloaded from UCSC Xena browser [46]. 
The dataset includes miRNA expression data from each sampled tumor, as well as corresponding demographic and clinical information such as patient survival and presence of metastases. For statistical analyses, expression values were $\log 2$ transformed to achieve a normal distribution. All statistical analyses were performed using the $\mathrm{R}$ language and environment for statistical computing ( $\mathrm{R}$ version 3.5.2; R Foundation for Statistical Computing; https:/www.r-project.org; RRID: SCR_001905).

\section{Differential expression analysis}

miRNA expression observations were normalized and differential miRNA expression between metastatic and nonmetastatic tumors was analyzed for all evaluable miRNAs in TCGA UM dataset using the LIMMA package (RRID:
SCR_010943) [47]. $P$-values were adjusted using the false discovery rate (FDR) method. Also, to minimize the false positives, a cut-off of fold change $>2$ and adj. $p$-value $<$ 0.01 was used to select the differentially expressed miRNAs.

\section{Survival analysis}

The survival difference between patients with vs. without metastatic UM in this TCGA dataset was calculated using the Cox proportional hazard model [48]. Independently, we performed survival analyses for each miRNA in the UM TCGA dataset. For each miRNA, subjects were separated into high-expression or lowexpression groups relative to the median expression value. Cox proportional hazard models were fitted for each miRNA. The $p$-values for HRs were computed and adjusted using the FDR method.

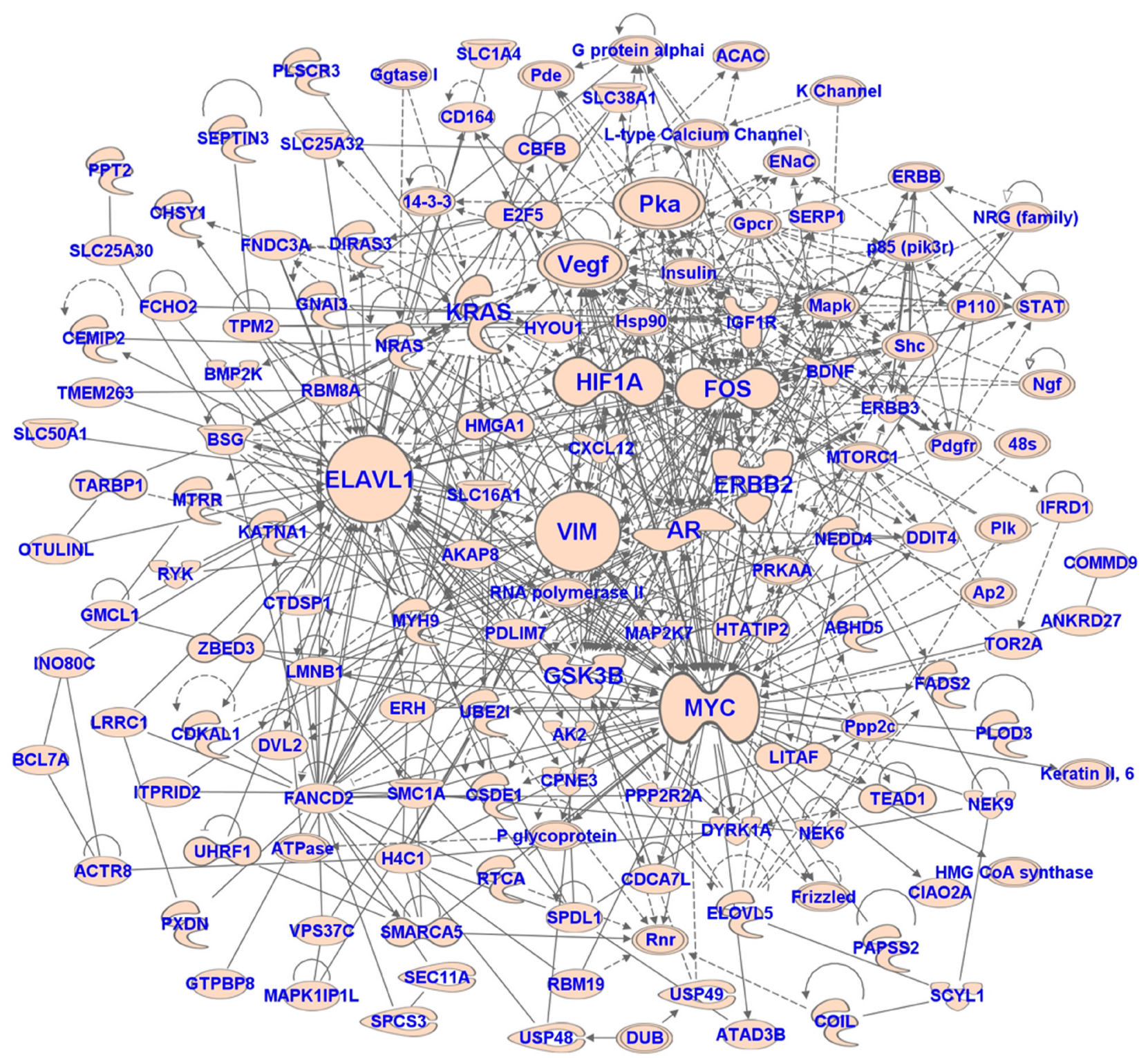

Figure 5: The network of target genes of miRNAs associated with metastasis in UM. The network was generated using IPA software. 


\section{Bioinformatics analyses}

Ingenuity Pathway Analysis (IPA) software was used to identify the target genes of miRNAs found to be associated with UM metastasis. Bioinformatics analyses of the target genes were performed using the IPA software for identification of enriched canonical pathways and biological functions. The prediction of upstream regulators was also done using the IPA software.

\section{Author contributions}

AV contributed to conceptualization. AV and TJL contributed to methodology, data curation, and formal analysis. AV, TJL, and JJW contributed review and interpretation of data analysis. AV, TJL, and JJW contributed to writing. AV, TJL, and JJW contributed to editing. AS contributed to bioinformatics analyses to identify gene targets and pathways. JJW contributed to final review and submission.

\section{CONFLICTS OF INTEREST}

The authors declare no relevant conflicts of interest.

\section{FUNDING}

JJW was supported in part by the Eunice Kennedy Shriver National Institute of Child Health and Human Development under award number K12HD085817. The content of this manuscript is solely the responsibility of the authors and does not necessarily represent the official views of the National Institutes of Health.

\section{REFERENCES}

1. Castet F, Garcia-Mulero S, Sanz-Pamplona R, Cuellar A, Casanovas O, Caminal JM, Piulats JM. Uveal Melanoma, Angiogenesis and Immunotherapy, Is There Any Hope? Cancers (Basel). 2019; 11:834. https://doi.org/10.3390/ cancers 11060834. [PubMed]

2. Souto EB, Zielinska A, Luis M, Carbone C, Martins-Gomes C, Souto SB, Silva AM. Uveal melanoma: physiopathology and new in situ-specific therapies. Cancer Chemother Pharmacol. 2019; 84:15-32. https://doi.org/10.1007/ s00280-019-03860-z. [PubMed]

3. Seider MI, Mruthyunjaya P. Molecular Prognostics for Uveal Melanoma. Retina. 2018; 38:211-219. https://doi. org/10.1097/IAE.0000000000001757. [PubMed]

4. Piperno-Neumann S, Piulats JM, Goebeler M, Galloway I, Lugowska I, Becker JC, Vihinen P, Van Calster J, Hadjistilianou T, Proença R, Caminal JM, Rogasik M, Blay JY, Kapiteijn E. Uveal Melanoma: A European Network to Face the Many Challenges of a Rare Cancer. Cancers (Basel). 2019; 11:817. https://doi.org/10.3390/cancers11060817. [PubMed]
5. Buder K, Gesierich A, Gelbrich G, Goebeler M. Systemic treatment of metastatic uveal melanoma: review of literature and future perspectives. Cancer Med. 2013; 2:674-686. https://doi.org/10.1002/cam4.133. [PubMed]

6. Augsburger JJ, Corrêa ZM, Shaikh AH. Effectiveness of treatments for metastatic uveal melanoma. Am J Ophthalmol. 2009; 148:119-127. https://doi.org/10.1016/j. ajo.2009.01.023. [PubMed]

7. Diener-West M, Reynolds SM, Agugliaro DJ, Caldwell R, Cumming K, Earle JD, Hawkins BS, Hayman JA, Jaiyesimi I, Jampol LM, Kirkwood JM, Koh WJ, Robertson DM, et al, and Collaborative Ocular Melanoma Study Group. Development of metastatic disease after enrollment in the COMS trials for treatment of choroidal melanoma: Collaborative Ocular Melanoma Study Group Report No. 26. Arch Ophthalmol. 2005; 123:1639-1643. https://doi. org/10.1001/archopht.123.12.1639. [PubMed]

8. Kaliki S, Shields CL, Shields JA. Uveal melanoma: estimating prognosis. Indian J Ophthalmol. 2015; 63:93102. https://doi.org/10.4103/0301-4738.154367. [PubMed]

9. Furney SJ, Pedersen M, Gentien D, Dumont AG, Rapinat A, Desjardins L, Turajlic S, Piperno-Neumann S, de la Grange P, Roman-Roman S, Stern MH, Marais R. SF3B1 mutations are associated with alternative splicing in uveal melanoma. Cancer Discov. 2013; 3:1122-1129. https://doi. org/10.1158/2159-8290.CD-13-0330. [PubMed]

10. Saldanha G, Purnell D, Fletcher A, Potter L, Gillies A, Pringle JH. High BRAF mutation frequency does not characterize all melanocytic tumor types. Int J Cancer. 2004; 111:705-710. https://doi.org/10.1002/ijc.20325. [PubMed]

11. Van Raamsdonk CD, Griewank KG, Crosby MB, Garrido MC, Vemula S, Wiesner T, Obenauf AC, Wackernagel W, Green G, Bouvier N, Sozen MM, Baimukanova G, Roy $\mathrm{R}$, et al. Mutations in GNA11 in uveal melanoma. N Engl J Med. 2010; 363:2191-2199. https://doi.org/10.1056/ NEJMoa1000584. [PubMed]

12. Zuidervaart W, van Nieuwpoort F, Stark M, Dijkman R, Packer L, Borgstein AM, Pavey S, van der Velden P, Out C, Jager MJ, Hayward NK, Gruis NA. Activation of the MAPK pathway is a common event in uveal melanomas although it rarely occurs through mutation of BRAF or RAS. Br J Cancer. 2005; 92:2032-2038. https://doi. org/10.1038/sj.bjc.6602598. [PubMed]

13. Horsman DE, White VA. Cytogenetic analysis of uveal melanoma. Consistent occurrence of monosomy 3 and trisomy 8q. Cancer. 1993; 71:811-819. https://doi. org/10.1002/1097-0142(19930201)71:3<811::AIDCNCR2820710325>3.0.CO;2-F. [PubMed]

14. Afshar AR, Damato BE, Stewart JM, Zablotska LB, Roy R, Olshen AB, Joseph NM, Bastian BC. Next-Generation Sequencing of Uveal Melanoma for Detection of Genetic Alterations Predicting Metastasis. Transl Vis Sci Technol. 2019; 8:18. https://doi.org/10.1167/tvst.8.2.18. [PubMed]

15. Zhou XG, Huang XL, Liang SY, Tang SM, Wu SK, Huang TT, Mo ZN, Wang QY. Identifying miRNA and gene 
modules of colon cancer associated with pathological stage by weighted gene co-expression network analysis. OncoTargets Ther. 2018; 11:2815-2830. https://doi. org/10.2147/OTT.S163891. [PubMed]

16. Liu M, Zhi Q, Wang W, Zhang Q, Fang T, Ma Q. Upregulation of miR-592 correlates with tumor progression and poor prognosis in patients with colorectal cancer. Biomed Pharmacother. 2015; 69:214-220. https://doi. org/10.1016/j.biopha.2014.12.001. [PubMed]

17. Mei LL, Wang WJ, Qiu YT, Xie XF, Bai J, Shi ZZ. miR$125 \mathrm{~b}-5 \mathrm{p}$ functions as a tumor suppressor gene partially by regulating HMGA2 in esophageal squamous cell carcinoma. PLoS One. 2017; 12:e0185636. https://doi.org/10.1371/ journal.pone.0185636. [PubMed]

18. Feng Y, Bai F, You Y, Bai F, Wu C, Xin R, Li X, Nie Y. Dysregulated microRNA expression profiles in gastric cancer cells with high peritoneal metastatic potential. Exp Ther Med. 2018; 16:4602-4608. https://doi.org/10.3892/ etm.2018.6783. [PubMed]

19. Zhou J, Liu R, Wang Y, Tang J, Tang S, Chen X, Xia K, Xiong W, Xu D, Wang S, He Q, Cao K. miR-199a-5p regulates the expression of metastasis-associated genes in B16F10 melanoma cells. Int J Clin Exp Pathol. 2014; 7:7182-7190. [PubMed]

20. Chu A, Robertson G, Brooks D, Mungall AJ, Birol I, Coope R, Ma Y, Jones S, Marra MA. Large-scale profiling of microRNAs for The Cancer Genome Atlas. Nucleic Acids Res. 2016; 44:e3. https://doi.org/10.1093/nar/gkv808. [PubMed]

21. Worley LA, Long MD, Onken MD, Harbour JW. Micro-RNAs associated with metastasis in uveal melanoma identified by multiplexed microarray profiling. Melanoma Res. 2008; 18:184-190. https://doi.org/10.1097/CMR.0b013e3282feeac6. [PubMed]

22. Venkatesan N, Kanwar J, Deepa PR, Khetan V, Crowley TM, Raguraman R, Sugneswari G, Rishi P, Natarajan V, Biswas J, Krishnakumar S. Clinico-Pathological Association of Delineated miRNAs in Uveal Melanoma with Monosomy 3/Disomy 3 Chromosomal Aberrations. PLoS One. 2016; 11:e0146128. https://doi.org/10.1371/ journal.pone.0146128. [PubMed]

23. Zhao G, Yin Y, Zhao B. miR-140-5p is negatively correlated with proliferation, invasion, and tumorigenesis in malignant melanoma by targeting SOX4 via the Wnt/beta-catenin and NF-kappaB cascades. J Cell Physiol. 2020; 235:2161-2170. https://doi.org/10.1002/jcp.29122. [PubMed]

24. Achberger S, Aldrich W, Tubbs R, Crabb JW, Singh AD, Triozzi PL. Circulating immune cell and microRNA in patients with uveal melanoma developing metastatic disease. Mol Immunol. 2014; 58:182-186. https://doi. org/10.1016/j.molimm.2013.11.018. [PubMed]

25. Russo A, Caltabiano R, Longo A, Avitabile T, Franco LM, Bonfiglio V, Puzzo L, Reibaldi M. Increased Levels of miRNA-146a in Serum and Histologic Samples of Patients with Uveal Melanoma. Front Pharmacol. 2016; 7:424. https://doi.org/10.3389/fphar.2016.00424. [PubMed]
26. Liu N, Sun Q, Chen J, Li J, Zeng Y, Zhai S, Li P, Wang B, Wang X. MicroRNA-9 suppresses uveal melanoma cell migration and invasion through the NF-kappaB1 pathway. Oncol Rep. 2012; 28:961-968. https://doi.org/10.3892/ or.2012.1905. [ubMed]

27. Venza I, Visalli M, Beninati C, Benfatto S, Teti D, Venza M. IL-10Ralpha expression is post-transcriptionally regulated by miR-15a, miR-185, and miR-211 in melanoma. BMC Med Genomics. 2015; 8:81. https://doi.org/10.1186/s12920015-0156-3. [PubMed]

28. Xia Z, Yang C, Yang X, Wu S, Feng Z, Qu L, Chen X, Liu L, Ma Y. miR-652 Promotes Proliferation and Migration of Uveal Melanoma Cells by Targeting HOXA9. Med Sci Monit. 2019; 25:8722-8732. https://doi.org/10.12659/ MSM.917099. [PubMed]

29. Sun L, Bian G, Meng Z, Dang G, Shi D, Mi S. MiR-144 Inhibits Uveal Melanoma Cell Proliferation and Invasion by Regulating c-Met Expression. PLoS One. 2015; 10:e0124428. https://doi.org/10.1371/journal.pone.0124428. [PubMed]

30. Yang C, Wei W. The miRNA expression profile of the uveal melanoma. Sci China Life Sci. 2011; 54:351-358. https:// doi.org/10.1007/s11427-011-4149-y. [PubMed]

31. Guo SJ, Zeng HX, Huang P, Wang S, Xie CH, Li SJ. MiR508-3p inhibits cell invasion and epithelial-mesenchymal transition by targeting ZEB1 in triple-negative breast cancer. Eur Rev Med Pharmacol Sci. 2018; 22:6379-6385. https://doi.org/10.26355/eurrev_201810 16050. [PubMed]

32. Zhai Q, Zhou L, Zhao C, Wan J, Yu Z, Guo X, Qin J, Chen $\mathrm{J}, \mathrm{Lu}$ R. Identification of miR-508-3p and miR-509-3p that are associated with cell invasion and migration and involved in the apoptosis of renal cell carcinoma. Biochem Biophys Res Commun. 2012; 419:621-626. https://doi. org/10.1016/j.bbrc.2012.02.060. [PubMed]

33. Chan CK, Pan Y, Nyberg K, Marra MA, Lim EL, Jones SJ, Maar D, Gibb EA, Gunaratne PH, Robertson AG, Rowat AC. Tumour-suppressor microRNAs regulate ovarian cancer cell physical properties and invasive behaviour. Open Biol. 2016; 6:160275. https://doi.org/10.1098/rsob.160275. [PubMed]

34. Coupland SE, Lake SL, Zeschnigk M, Damato BE. Molecular pathology of uveal melanoma. Eye (Lond). 2013; 27:230-242. https://doi.org/10.1038/eye.2012.255. [PubMed]

35. Kim YJ, Park SJ, Maeng KJ, Lee SC, Lee CS. MultiPlatform Omics Analysis for Identification of Molecular Characteristics and Therapeutic Targets of Uveal Melanoma. Sci Rep. 2019; 9:19235. https://doi.org/10.1038/ s41598-019-55513-Z. [PubMed]

36. Monroig PC, Chen L, Zhang S, Calin GA. Small molecule compounds targeting miRNAs for cancer therapy. Adv Drug Deliv Rev. 2015; 81:104-116. https://doi.org/10.1016/j. addr.2014.09.002. [PubMed]

37. Krutzfeldt J, Rajewsky N, Braich R, Rajeev KG, Tuschl T, Manoharan M, Stoffel M. Silencing of microRNAs in vivo with 'antagomirs'. Nature. 2005; 438:685-689. https://doi. org/10.1038/nature04303. [ [ 
38. Elmen J, Lindow M, Schutz S, Lawrence M, Petri A, Obad S, Lindholm M, Hedtjarn M, Hansen HF, Berger U, Gullans S, Kearney P, Sarnow P, et al. LNA-mediated microRNA silencing in non-human primates. Nature. 2008; 452:896899. https://doi.org/10.1038/nature06783. [PubMed]

39. Janssen HL, Reesink HW, Lawitz EJ, Zeuzem S, RodriguezTorres M, Patel K, van der Meer AJ, Patick AK, Chen A, Zhou Y, Persson R, King BD, Kauppinen S, et al. Treatment of HCV infection by targeting microRNA. N Engl J Med. 2013; 368:1685-1694. https://doi.org/10.1056/ NEJMoa1209026. [PubMed]

40. Ebert MS, Neilson JR, Sharp PA. MicroRNA sponges: competitive inhibitors of small RNAs in mammalian cells. Nat Methods. 2007; 4:721-726. https://doi.org/10.1038/ nmeth1079. [PubMed]

41. Gaur S, Wen Y, Song JH, Parikh NU, Mangala LS, Blessing AM, Ivan C, Wu SY, Varkaris A, Shi Y, Lopez-Berestein G, Frigo DE, Sood AK, Gallick GE. Chitosan nanoparticlemediated delivery of miRNA-34a decreases prostate tumor growth in the bone and its expression induces non-canonical autophagy. Oncotarget. 2015; 6:29161-29177. https://doi. org/10.18632/oncotarget.4971. [PubMed]

42. Bonci D, Coppola V, Musumeci M, Addario A, Giuffrida R, Memeo L, D’Urso L, Pagliuca A, Biffoni M, Labbaye C, Bartucci M, Muto G, Peschle C, De Maria R. The miR15a-miR-16-1 cluster controls prostate cancer by targeting multiple oncogenic activities. Nat Med. 2008; 14:12711277. https://doi.org/10.1038/nm.1880. [PubMed]

43. Trang P, Medina PP, Wiggins JF, Ruffino L, Kelnar K, Omotola M, Homer R, Brown D, Bader AG, Weidhaas JB, Slack FJ. Regression of murine lung tumors by the let-7 microRNA. Oncogene. 2010; 29:1580-1587. https://doi. org/10.1038/onc.2009.445. [PubMed]
44. Esposito CL, Cerchia L, Catuogno S, De Vita G, Dassie JP, Santamaria G, Swiderski P, Condorelli G, Giangrande $\mathrm{PH}$, de Franciscis V. Multifunctional aptamer-miRNA conjugates for targeted cancer therapy. Mol Ther. 2014; 22:1151-1163. https://doi.org/10.1038/mt.2014.5. [PubMed]

45. Reid G, Kao SC, Pavlakis N, Brahmbhatt H, MacDiarmid J, Clarke S, Boyer M, van Zandwijk N. Clinical development of TargomiRs, a miRNA mimic-based treatment for patients with recurrent thoracic cancer. Epigenomics. 2016; 8:10791085. https://doi.org/10.2217/epi-2016-0035. [PubMed]

46. Goldman M, Craft B, Hastie M, Repečka K, Kamath A, McDade F, Rogers D, Brooks AN, Zhu J, Haussler D. The UCSC Xena platform for public and private cancer genomics data visualization and interpretation. bioRxiv. 2019; 326470. https://doi.org/10.1101/326470.

47. Ritchie ME, Phipson B, Wu D, Hu Y, Law CW, Shi W, Smyth GK. limma powers differential expression analyses for RNA-sequencing and microarray studies. Nucleic Acids Res. 2015; 43:e47. https://doi.org/10.1093/nar/gkv007. [PubMed]

48. Cox DR. Regression models and life-tables. J R Stat Soc B. 1972; 34:187-202. https://doi.org/10.1111/j.2517-6161.1972. tb00899.x. 